Research Square

\title{
On the mathematical lifetime mechanism of Chinese dynasties
}

\section{Yufei Cao ( $\nabla$ caoyufeiluo@126.com )}

Shanghai Lixin University of Accounting and Finance https://orcid.org/0000-0003-1325-8484

\section{Research Article}

Keywords: continuous-time Markov chains, Coxian phase-type distributions, transition probabilities, survival function, Chinese dynasties, social states

Posted Date: March 1st, 2022

DOI: https://doi.org/10.21203/rs.3.rs-1128370/v1

License: (c) (i) This work is licensed under a Creative Commons Attribution 4.0 International License. Read Full License 


\title{
On the mathematical lifetime mechanism of Chinese dynasties
}

\begin{abstract}
The long history of China is composed of different imperial dynasties. However, all dynasties only survive limited time and follow the identical development process, which is a dynamical process from establishment to extinction. Based on the historical fact about the Chinese dynasties, this paper proposes a continuous-time Markov chain model combined with Coxian phase-type distributions to investigate the survival time for Chinese dynasties. Our model includes four different states, which correspond to the four typical social and political states for the traditional Chinese imperial dynasties. Three states are transient, which are the establishment state, the prosperity state, the rottenness state, and one state is absorbing, which is the perishment state. We use the historical survival time data for Chinese dynasties to estimate our model by maximizing the log-likelihood function. We obtain the closed-forms for the transition probabilities, the survival function and the force of mortality for Chinese dynasties. The analysis for hitting times and absorption probabilities shows that Chinese dynasties can survive 92 years on average. We use our model to conduct the phases analysis, which can be used to predict the current social state given the excising survival years for Chinese dynasties. Based on our analysis, we give suggestions on foreign policy and business.
\end{abstract}

Keywords: continuous-time Markov chains, Coxian phase-type distributions, transition probabilities, survival function, Chinese dynasties, social states

\section{Introduction}

The empire, long divided, must unite; long united, must divide. Thus it has ever been. In the closing years of the Zhou dynasty, seven kingdoms warred among themselves until the kingdom of Qin prevailed and absorbed the other six. But Qin soon fell, and on its ruins two opposing kingdoms, Chu and Han, fought for mastery until the kingdom of Han prevailed and absorbed its rival, as Qin had done before.

Three Kingdoms: A Historical Novel, Luo (2004).

The above sentence is the beginning of the famous historical novel in China, Three Kingdoms: $A$ Historical Novel, and plays a critical role to understand the philosophy and social status of China. In particular, this sentence indicates the historical and political philosophy of the Chinese people in some extent and may be considered as a miniature version of the mechanism of Chinese dynasties. The long history of China is composed of various and successive imperial dynasties. Dynastic change is a wellknown aspect for the Chinese history. The rise and fall of Chinese dynasties constitutes the dynamical development process of China. Traditional imperial Chinese dynasties are often established by families or clans through countless cruel wars. However, the history of China shows that the development for all 
these dynasties follows an identical pattern, which may be regarded as a cyclical birth and death process. This pattern is mainly caused by the fact that the Chinese dynasties are always powerful centralized countries, implying that only the emperor has the supreme power.

A classical developing process for Chinese dynasties is illustrated as follows. In Chinese history, when a dynasty is established at the early stage, its politic environment is often clear and open. The emperor focuses on economic recovery and development. As a result, the social stability and national strength of the dynasty has been enhanced, which is sometimes often called as political and economic resurgence by Chinese historians. However, after a long period of rapid development, Chinese dynasties often tend to political and economic corruption, which usually leads to perish, because of the weakness of the imperial power system. That is, there is no power supervision system for the imperial power. Furthermore, when a dynasty tends to perish, it constantly encounters the uprising wars of Chinese peasants and the imperial autocratic power system often is full of political and economic corruptions. The Chinese history shows that the result of these uprising wars is that a new imperial dynasty is constructed and the old one is perished. Then a new dynastical cycle begins. All the Chinese dynasties are built on the ruins of the previous one.

The cyclical pattern described above shows that a traditional Chinese imperial dynasty is constructed first, and after a period of development it eventually evolves into the uprising wars of Chinese peasants, which make the dynasty be destroyed. There exists 70 different dynasties in total in the history of China, which is listed later. The change of imperial dynasties indicates that all these traditional imperial dynasties in China only can survive finite time. However, more interestingly, all historical Chinese autocratic emperors dreamed of long live in order to sustain their dynasties and maintain the benefit of power. Motivated by the historical fact that every Chinese dynasty only survive finite time, this article proposes a mathematical model to investigate the lifetime mechanism for Chinese dynasties. For policymakers and business companies, our model is helpful to analyze the social state of Chinese dynasties and provides information on policy affairs and decisions with China.

The reason why the above dynastic cycle ceaselessly repeated in the Chinese history has been extensively studied from various aspects. Early work (Usher (1989)) divides the Chinese population into farmers, bandits and rulers and claims that the condition of farmers has impact on the social state, which is either in despotism or anarchy. Zhang (2015) argues that the change of dynasties is caused by the primacy of public will and that the rise or fall of any dynasty depends on the support of the people. The review paper by wai Fan (2009) claims that climatic change also had significant impact on the rise and fall of Chinese dynasties and that the fall of the Tang and Ming dynasties may have been caused by global cooling. Another perspective to study the dynastic cycles is to model the Chinese population, which is often divided into farmers, bandits and rules. If there is shortage of food, farmers turned into bandits, then there are wars against the rulers (Yu-Ch'uan (1936), Skinner (1985)). The population model is extended by adding economic assumption of utility maximization, see, Chu and Lee (1994) and Chan and Laffargue (2016).

In this paper, we argues that the history of China constantly follows and repeats the dynastic cycle mentioned above, because Chinese dynasties are political power centralized and follows the imperial 
autocracy political power system. This political system itself always leads to institutional and systemic corruptions when the dynasties develop in depth. These corruptions eventually generate serious economic crisis and social turbulence. In traditional Chinese imperial dynasties, the political power determines every aspect of the society. The development of the traditional Chinese dynasties may be regard as a dynamic process, whose main aim is to strengthen the imperial autocracy political power for the emperor. The fundamental characteristic of the imperial autocratic political power system is that the emperor is the most important in the dynasty. In order to ensure the emperors and their generations own corruption privileges, the emperors establish a huge bureaucratic system and try to control every aspect of the society. The entire country, people and bureaucratic groups only serve for the emperor, not on the contrary. The whole dynasty in the ancient China is governed by the power of the emperor and the Chinese history shows that the political power entirely depends on the violence. Because the imperial autocracy political power rules everything in the traditional China, the political power is not bound and the usage of the power is arbitrary. That is, the power of the emperor is not supervised and rejects civilian supervision. This drawback of the imperial autocracy political power system is the main reason causing that dynasties follows a raise and fall pattern. Moreover, the Chinese history shows that such power system always generates sever economic problems. The development of Chinese dynasties often shows that after a long period of development, the emperors become self-satisfied and gradually start to waste the country's tax, on traveling, building palaces, and distributing money to foreign countries. As a consequence, people living in the traditional dynasties have to continuously face the heavy tax collection, and then start to challenge the dynasty through wars. Hence, for policy-makers and foreign business companies, it becomes easily to promote business with those who have the superior power and influence.

In this paper, we assume that the dynamical development process for the Chinese dynasties are persistent, because the long history of China shows that almost all dynasties followed the power centralization political system. Motivated by the cyclical birth and death development pattern of the imperial Chinese dynasties, we propose a continuous-time Markov chain to model the social development states and survival time for the Chinese dynasties. There are extensive researches on explaining the developing process for China, but, to the authors' best knowledge, our proposed model is the first one to mathematically investigate the developing social process and the surviving time for the Chinese dynasties. Based on the development of each dynasty from the Chinese history, our proposed Markov chain has four different states, which are the establishment state, the prosperity state, the rottenness state and the perishment state. These four political and social states characterize the feature of Chinese dynasties and the power centralization political system. Our model starts from the establishment state, because the Chinese dynasty has to establish at first. The first three states are transient, and only the perishment state is absorbed. In our model, we assume that the three transient states are ordered and any transient states can directly jump into the perishemnt state. That is, the transient states transfer either into the next transient state or directly into the absorbing state. Generally speaking, the Chinese often assumes that the development for Chinese dynasties is usually linear. Namely, one dynasty starts from the establishment state, then moves into the prosperity state; after the prosperity state, the dynasty enters into the rottenness state and then finally to the perishement state. When the dynasty is in the perish- 
ment state, it means that the dynasty perishes and a new dynasty is established. The transition rule embedded in the continuous-time Markov chain indicates that the surviving time of the dynasty follows a Coxian phase-type distribution. The advantage of using the Coxian phase-type distribution is that the probability density function can be expressed in the closed form. Coxian phase-type distributions are a special case of phase-type distributions. Phase-type distributions have been widely used in the stochastic modelling applications, such as biostatistics, queueing theory, risk theory, reliability theory and survival analysis (see, for example, Hobolth et al. (2019), Bladt (2005), Neuts (1981), Payne et al. (2011), Govorun et al. (2018) and Okamura and Dohi (2016)).

We use hand-collected surviving time data for each dynasty to approximate our continuous-time Markov chain model. Our model is estimated by maximizing the log-likelihood function. To analyze our estimated model, we consider five different variables of interest, which are closely related with the development states and the survival time data for Chinese dynasties. The first variable is to measure the transition probabilities between four different social states. These transition probabilities are useful to determine the state transition for the dynasty. Then, we consider hitting times and absorption probabilities for the Chinese dynasties. By computing hitting time, we are able to find the average surviving time for Chinese dynasties. The empirical results show that on average, it takes about 92 years from the establishment state to the perishment state for Chinese dynasties, which is useful to study the Chinese history and its future development direction. More interestingly, our model indicates that every dynasty in China is destined to perish. The third variable examines the survival function, which is used to estimates the probability that Chinese dynasties can survive after existing a certain time period. Fourth, we define a conditional probability which is used to identify the present state of Chinese dynasties. This conditional probability is used to compute the probability that the dynasty is at a specific social state conditional on that the dynasty has lived longer than a certain time period. Finally, we calculate the force of mortality for Chinese dynasties, which can be used to determine the instant death rate at a certain time. All these variables evaluated above are expressed in the closedforms. Foreign policy-makers and business companies may find that these variables are helpful to make proper policies and investment strategies.

The rest of this paper is organized as follows. Section 2 introduces the mathematical method. Section 3 presents the proposed lifetime model for the Chinese dynasties. Section 4 lists the dynasties' names appeared in the Chinese history and shows their survival time data. Section 5 shows the empirical results and analyzes the five different variables of interest, which may be used to predict the development states for Chinese dynasties. Finally, Section 6 concludes and presents the future work.

\section{Basic mathematics}

One aim of this article is to determine the probability distribution of the surviving time for an imperial Chinese dynasty. Our proposed model is related with Coxian phase-type distributions. Hence, this section first briefly introduces the definition of the general phase-type distributions, and then presents the Coxian phase-type distributions. For a comprehensive theoretical treatment and applications of phase-type distributions, see, for example, Neuts (1981), Neuts (1991) and Asmussen (2003). 


\subsection{Phase-type distributions}

Given a continuous-time Markov chain with $n$ transient states (or phases) and a single absorbing state, a continuous random variable is said to be a phase-type distribution if this random variable determines the probability distribution of the time to absorption. The mathematical properties of phasetype distributions have been studied extensively. Their density probability functions, survival functions and moments can be expressed by simple analytical closed-forms in terms of matrix exponentials. We present their basic properties as follows.

Let $\{X(t) ; t \geq 0\}$ be a continuous-time Markov chain with $n+1$ states, where states $1,2, \cdots, n$ are transient and state $n+1$ is absorbing. To construct a continuous-time Markov chain requires two building blocks (see Norris (1997)). One block is the initial distribution, and the other block is the infinitesimal generator matrix, which determines how the process evolves from its initial state. For the first block, let $X(t)$ have the initial distribution $(\boldsymbol{\alpha}, 0)$, where $\boldsymbol{\alpha}$ is a $1 \times n$ row vector. The initial distribution indicates that the continuous-time Markov process starts from one of the transient states. For the second block, let the infinitesimal generator matrix $\boldsymbol{Q}$ of $X(t)$ have the following form:

$$
Q=\left(\begin{array}{ll}
\Lambda & q \\
0 & 0
\end{array}\right),
$$

where $\boldsymbol{\Lambda}$ is the $n \times n$ transition rate matrix and describes the transition rates among transient states, and $\boldsymbol{q}$ is the $n \times 1$ column absorbing rate vector and determines the transition rates from the transient states to the absorbing state. Note that the row sum of $\boldsymbol{Q}$ must be equal to zero, namely, $\boldsymbol{q}=-\boldsymbol{\Lambda} \boldsymbol{e}$, where $\boldsymbol{e}$ is the column vector of ones.

Let the random variable $T$ denote the time until absorption for the underlying continuous-time Markov chain $X(t)$. Then, $T$ is said to follow a phase-type distribution with representation $(\boldsymbol{\alpha}, \boldsymbol{\Lambda})$.

Phase-type distributions are attractive for applications, because they provide the intuitive interpretation for the mechanism of the system, whose property is related with the lifetime survival distributions. That is, the survival probability of the system at any time is the sum of the probability that the process is in any transient states before it enters into the absorbing state.

Many mathematical properties of the phase-type distributions can be expressed in the closed-forms. The probability density function of the random variable $T$ is

$$
f(t)=\boldsymbol{\alpha} \exp (\boldsymbol{\Lambda} t) \boldsymbol{q}
$$

where $t \geq 0$. Here, the matrix exponential $\exp (\boldsymbol{\Lambda} t)$ is defined as (see Higham (2008))

$$
\exp (\boldsymbol{\Lambda} t)=\sum_{n=0}^{\infty} \frac{t^{n}}{n !} \mathbf{\Lambda}^{n}
$$

where $\boldsymbol{\Lambda}^{0}=\boldsymbol{I}$. The cumulative distribution function $F(t)$ of the random variable $T$ is given by

$$
F(t)=1-\boldsymbol{\alpha} \exp (\boldsymbol{\Lambda} t) \boldsymbol{e}
$$

and the survival function $S(t)$ of the random variable $T$ is

$$
S(t)=\boldsymbol{\alpha} \exp (\boldsymbol{\Lambda} t) \boldsymbol{e}
$$


Phase-type distributions have concise matrix expressions. However, in practical applications, computing the matrix exponentials presented in Equations (2), (3) and (4) is not trivial, especially when the dimension of $\boldsymbol{\Lambda}$ (i.e., the number of state, $n$ ) is large. The generality of the phase-type distributions makes parameters estimation difficult. Therefore, Coxian phase-type distributions are introduced.

\subsection{Coxian phase-type distributions}

The Coxian phase-type distributions (see Cox (1955)) are a special case of the general phase-type distributions, and their density probability functions, cumulative distribution functions and survival functions can also be expressed by Equations (2), (3) and (4), respectively. By comparing with the general phase-type distributions, the difference is that the transient states of the underlying continuoustime Markov chain are ordered. To form Coxian phase-type distributions, the continuous-time Markov chain must start from the first phase, and then may either move into the next phase or directly enter into the absorbing state. The benefit of this ordered structure is the reduction in the number of parameters in the infinitesimal generator matrix $\boldsymbol{Q}$. However, for a large number of phases, estimating the Coxian phase-type distributions still remain problematic (Faddy (1998)).

For the Coxian phase-type distributions, the initial distribution of the underlying continuous-time Markov chain $X(t)$ is written as

$$
\boldsymbol{\alpha}=(1, \underbrace{0, \cdots, 0}_{n-1})
$$

which indicates that $X(t)$ must start from the first transient state 1. Furthermore, the evolution process of $X(t)$ can be illustrated in Figure 1. All parameters $\lambda_{i}>0$ and $\mu_{i}>0$. The parameters $\lambda_{1}, \lambda_{2}, \cdots, \lambda_{n-1}$ represent the transition rates from the transient phase 1 to the phase 2 , the phase 2 to the phase 3 , and the phase $n-1$ to the phase $n$, respectively. The parameters $\mu_{1}, \mu_{2}, \cdots, \mu_{n}$ denote the transition rates from any phases $1,2, \cdots, n$ to the absorbing state $n+1$, respectively.

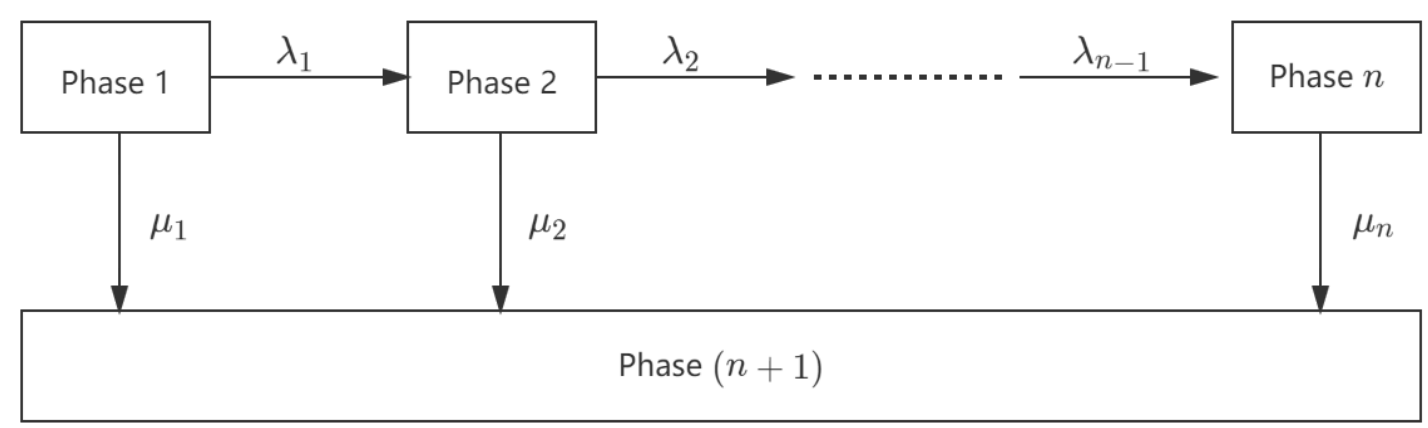

Figure 1: An illustration of Coxian phase-type distributions.

Figure 1 implies that the continuous-time Markov chain $X(t)$ has a specific infinitesimal generator 
matrix $\boldsymbol{Q}$, which has the transition rate matrix

$$
\boldsymbol{\Lambda}=\left(\begin{array}{ccccc}
-\left(\lambda_{1}+\mu_{1}\right) & \lambda_{1} & 0 & \ldots & 0 \\
0 & -\left(\lambda_{2}+\mu_{2}\right) & \lambda_{2} & \ldots & 0 \\
\vdots & \ddots & \ddots & \vdots & \vdots \\
0 & 0 & \ddots & -\left(\lambda_{n-1}+\mu_{n-1}\right) & \lambda_{n-1} \\
0 & 0 & \cdots & 0 & -\mu_{n}
\end{array}\right)
$$

and the absorbtion rate vector

$$
\boldsymbol{q}=\left(\mu_{1}, \mu_{2}, \cdots, \mu_{n}\right)^{\top} .
$$

Then, we can obtain the probability density function, the cumulative distribution function and the survival function for the Coxian phase-type distributions by inserting Equations (5), (6) and (7) into Equation (2), (3) and (4), respectively. When the dimension of the Coxian phase-type distributions is small, the transition rates in the Coxian model may be quickly evaluated by maximizing the log-likelihood function.

\subsection{Estimation}

The parameters $\lambda_{i}$ and $\mu_{i}$ appeared in the Coxian phase-type distributions can be estimated by maximizing the corresponding $\log$-likelihood function. Let $\boldsymbol{\theta}=\left(\lambda_{i}, \mu_{i}\right)$ be the parameter vector in the transition matrix $\boldsymbol{\Lambda}$. The log-likelihood function for the Coxian phase-type distribution is expressed as

$$
L(\boldsymbol{\theta})=\sum_{i=1}^{n} \log \left(\boldsymbol{\alpha} \exp \left(\boldsymbol{\Lambda} t_{i}\right) \boldsymbol{q}\right),
$$

where $\boldsymbol{\alpha}, \boldsymbol{\Lambda}$ and $\boldsymbol{q}$ are given by Equations (5), (6), and (7), respectively. Then, the log-likelihood function $L(\boldsymbol{\theta})$ is maximized numerically with respect to the parameters.

\section{Lifetime mechanism for Chinese dynasties}

This article focuses on mathematically modelling the lifetime mechanism of Chinese dynasties. We define the lifetime mechanism of Chinese dynasties as a dynamic developing process from the establishment to the perishment. The only difference lies in that dynasties are often founded by different clans or families. The Chinese history shows that every dynasty repeats this dynamic developing pattern, which is supported by the data of Chinese dynasties shown in the next section. In this paper, we consider the lifetime mechanism of Chinese dynasties in a Markovian environment with different social states, because the history of China persistently reveals that each dynasty presents significant social state transitions in its existing time period. In particular, we propose a Coxian phase-type distribution associated with a continuous-time Markov chain to determine the lifetime mechanism of Chinese dynasties. Our model provides an appealing approach to investigating the lifetime mechanism, because each Chinese dynasty survives a limited lifetime from its establishment to its demise and always tends to repeat a similar birth and death development pattern. Furthermore, the Coxian phase-type distributions have closed-forms for the density probability function and the survival function, which indicates that we can perform a variety 
of analyses to compute quantities of interest, such as the survival probability and the mean survival time for a Chinese dynasty.

Our approach to modelling the lifetime mechanism of Chinese dynasties is motivated by the properties of Coxian phase-type distributions. We model the lifetime mechanism by considering the diagram shown in Figure 2. This model is summarized by reviewing the history data of Chinese dynasties. In the history of China, almost all dynasties are build based on the ideology of centralization of power. As a consequence, these dynasties are highly bureaucratic inertia and are administered by a hierarchy of officials, indicating that their future follow a similar dynastic cycle of rise and fall. Figure 2 illustrates that the lifetime mechanism of a typical Chinese dynasty which consists of four different development phases (or social states): establishment, prosperity, rottenness and perishment. The establishment phase indicates that the dynasty is established at first. At any time thereafter, the dynasty may prosper or perish, which depends on the transition rates. If the dynasty is able to survive and enter into the prosperity state, the dynasty may either become rotten or perish. In Chinese history, there are so many rottenness social states after the dynasty luckily prospers, which may indicate the weakness and backwards of the political system of the traditional Chinese imperial dynasties. The rottenness phase shows that the dynasty will eventually perish. The traditional Chinese dynasties continuously repeat the transition pattern shown in Figure 2, because the Chinese history includes many different dynasties, which is seen in the next section. To approximate the lifetime mechanism for Chinese dynasties shown in Figure 2, we use the surviving data of Chinese dynasties.

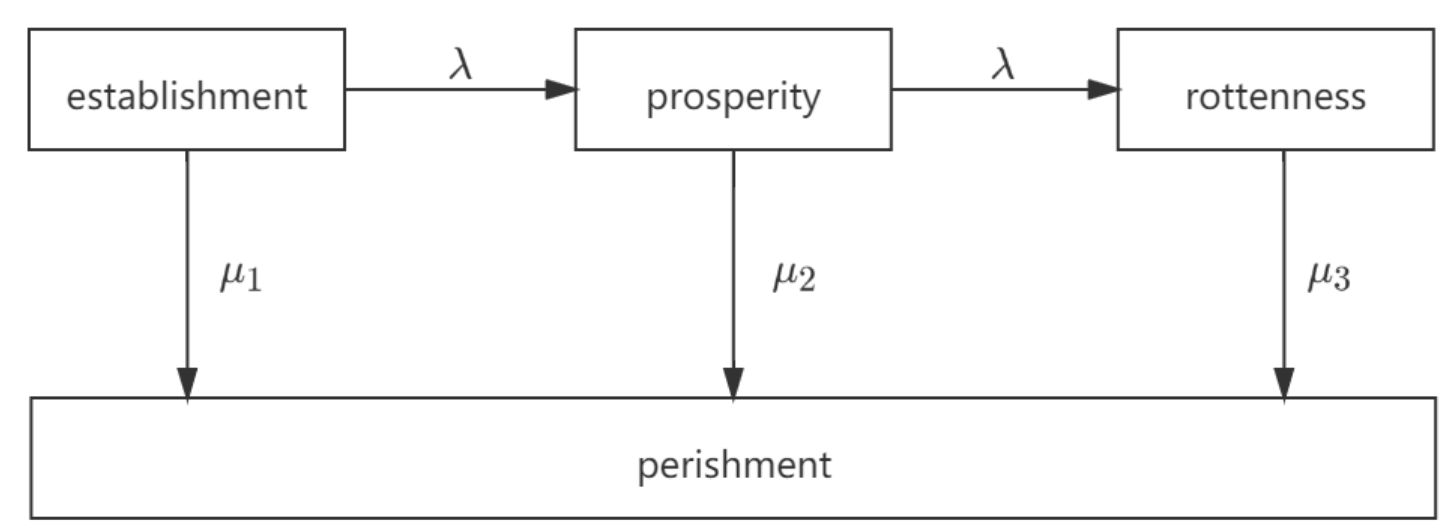

Figure 2: Diagram for the Coxian phase-type model for modelling the lifetime mechanism for Chinese dynasties.

We use similar mathematical notations in the previous section to describe the Coxian phase-type model illustrated in Figure 2. Let $\{X(t), t \geq 0\}$ be a continuous-time Markov chain, representing the phase or the social state of Chinese dynasties. In our model, we assume that the dynamical process $X(t)$ consists of four different phases, which correspond to four different social states as shown in Figure 2. Then, following the set-up procedures of the Coxian phase-type model described above, the initial 
distribution for the process $X(t)$ is given as

$$
\boldsymbol{\alpha}=(1,0,0)
$$

which indicates that the dynasty must begin from the establishment phase. Furthermore, the infinitesimal generator matrix $\boldsymbol{Q}$ for the process $X(t)$ is of a $4 \times 4$ matrix and can be written as

$$
\boldsymbol{Q}=\left(\begin{array}{ccc|c}
-\left(\lambda+\mu_{1}\right) & \lambda & 0 & \mu_{1} \\
0 & -\left(\lambda+\mu_{2}\right) & \lambda & \mu_{2} \\
0 & 0 & -\mu_{3} & \mu_{3} \\
\hline 0 & 0 & 0 & 0
\end{array}\right)
$$

Let the random variable $T$ denote the time from the establishment of the dynasty to its perishment for our proposed dynasty model, then $T$ follows the Coxian phase-type distribution with the representation $(\boldsymbol{\alpha}, \boldsymbol{\Lambda})$. The initial distribution $\boldsymbol{\alpha}$ is given by Equation (9). The transition rate matrix $\boldsymbol{\Lambda}$ is embedded in Equation (10) and has the following form

$$
\boldsymbol{\Lambda}=\left(\begin{array}{ccc}
-\left(\lambda+\mu_{1}\right) & \lambda & 0 \\
0 & -\left(\lambda+\mu_{2}\right) & \lambda \\
0 & 0 & -\mu_{3}
\end{array}\right)
$$

Moreover, the absorbtion rate vector $\boldsymbol{q}$ can also be obtained from Equation (10) and is given by

$$
\boldsymbol{q}=\left(\mu_{1}, \mu_{2}, \mu_{3}\right)^{\mathrm{T}}
$$

It is commonly known that the industrial revolution heavily had modified the West. However, the Chinese history shows that traditional Chinese imperial dynasties had long been big agricultural countries for almost all centuries and their prime social productivity was very low, implying that the process of social development is relatively stable. In this paper we suppose that the development of each dynasty keeps consistent with each other. Hence, we assume that the transition rate from the establishment phase to the prosperity phase is equal to the rate from the prosperity phase to the rottenness rate, which is denoted by $\lambda$ shown in Figure 2. Moreover, under this assumption, the empirical results are in agreement with the surviving time data for Chinese dynasties, which is seen later. To estimate our proposed dynasty model, inserting Equations (9), (11) and (12) into Equation (2) gives the closed-form for the probability density function of the Coxian phase-type random variable $T$. Then, the parameter vector $\boldsymbol{\theta}=\left(\lambda, \mu_{1}, \mu_{2}, \mu_{3}\right)$ is estimated by maximizing the corresponding log-likelihood function.

\section{Data and results}

\subsection{Data}

To estimate the Coxian phase-type model for the Chinese dynasties, proposed in Figure 2, we collect the survival time data for all the dynasties in the Chinese history. Tables 1 and 2 show all the Chinese dynasties and their survival time data. There are 70 different dynasties in China, implying that the long history of China is composed of various dynasties. More interestingly, almost all these dynasties 
follow the identical political system, which is centralization of power and started from Qin dynasty. This historical fact indicates that there is significant difference in political system in the East and West and that the developing process of the Chinese dynasties has persistence and stability. Since Qin dynasty, there is no political innovation and emperors of all dynasties only consider how to utilize and strengthen the centralization of power system. Hence, the history of China suggests that each dynasty follows and repeats a similar development and organization pattern, which is modelled by the Coxian phase-type model shown in Figure 2 in this article. Most dynasties are presented in the chronological order, while a few dynasties co-exist at the same time. The survival time date of the dynasties is computed from the difference between the start and the end year. We find that some dynasties were able to exist more than one hundred years, while others became extinct less than ten years. The survival data shows that Shang dynasty survived the longest time, which was 646 years, and Xia dynasty was the second longest survival dynasty, which lasted 471 years. There are few dynasties living beyond 300 years. However, most Chinese dynasties perish within 100 years. To sum up, the survival data implies that each dynasty only survives a limited time period and the political system never changes since Qin dynasty. 
Table 1: First part for Chinese dynasties and their survival time data.

\begin{tabular}{|c|c|c|c|c|}
\hline Rank & Dynasty name & Start & End & Duration (years) \\
\hline 1 & Xia & $2146 \mathrm{BC}$ & $1675 \mathrm{BC}$ & 471 \\
\hline 2 & Shang & $1675 \mathrm{BC}$ & $1029 \mathrm{BC}$ & 646 \\
\hline 3 & Xi Zhou & $1029 \mathrm{BC}$ & $771 \mathrm{BC}$ & 258 \\
\hline 4 & Chun Qiu & $770 \mathrm{BC}$ & $476 \mathrm{BC}$ & 294 \\
\hline 5 & Zhan Guo & $475 \mathrm{BC}$ & $221 \mathrm{BC}$ & 254 \\
\hline 6 & Qin & $221 \mathrm{BC}$ & $207 \mathrm{BC}$ & 14 \\
\hline 7 & Xi Han & $206 \mathrm{BC}$ & $8 \mathrm{AD}$ & 218 \\
\hline 8 & Xin Chao & 9 & 23 & 14 \\
\hline 9 & Dong Han & 25 & 220 & 195 \\
\hline 10 & Cao Wei & 220 & 265 & 45 \\
\hline 11 & Shu Han & 221 & 263 & 42 \\
\hline 12 & Sun Wu & 222 & 280 & 58 \\
\hline 13 & Xi Jin & 265 & 316 & 51 \\
\hline 14 & Dong Jin & 317 & 420 & 103 \\
\hline 15 & Qian Zhao & 304 & 318 & 14 \\
\hline 16 & Qian Zhao & 319 & 329 & 10 \\
\hline 17 & Cheng Han & 306 & 347 & 41 \\
\hline 18 & Qian Liang & 314 & 363 & 49 \\
\hline 19 & Hou Zhao & 319 & 351 & 32 \\
\hline 20 & Qian Yan & 337 & 370 & 33 \\
\hline 21 & Qian Qin & 351 & 394 & 43 \\
\hline 22 & Hou Qin & 384 & 417 & 33 \\
\hline 23 & Hou Yan & 384 & 407 & 23 \\
\hline 24 & Xi Qin & 385 & 431 & 46 \\
\hline 25 & Hou Liang & 386 & 403 & 17 \\
\hline 26 & Nan Liang & 397 & 414 & 17 \\
\hline 27 & Nan Yan & 398 & 410 & 12 \\
\hline 28 & Xi Liang & 407 & 421 & 14 \\
\hline 29 & $\mathrm{Hu}$ Xia & 407 & 431 & 24 \\
\hline 30 & Bei Yan & 407 & 436 & 29 \\
\hline 31 & Bei Liang & 397 & 439 & 42 \\
\hline 32 & Ran Wei & 350 & 352 & 2 \\
\hline 33 & Xi Yan & 384 & 394 & 10 \\
\hline 34 & Hou Shu & 405 & 413 & 8 \\
\hline 35 & Song & 420 & 479 & 59 \\
\hline
\end{tabular}

Notes: The survival time data is obtained from the difference between the start and end year. The bold number shows the longest surviving time data in China. This table is the first part of all Chinese dynasties. 
Table 2: Second part for Chinese dynasties and their survival time data.

\begin{tabular}{|c|c|c|c|c|}
\hline Rank & Dynasty name & Start & End & Duration (years) \\
\hline 36 & Qi & 479 & 502 & 23 \\
\hline 37 & Liang & 502 & 557 & 55 \\
\hline 38 & Chen & 557 & 589 & 32 \\
\hline 39 & Bei Wei & 386 & 534 & 148 \\
\hline 40 & Dong Wei & 534 & 550 & 16 \\
\hline 41 & Xi Wei & 535 & 556 & 21 \\
\hline 42 & Bei Qi & 550 & 577 & 27 \\
\hline 43 & Bei Zhou & 557 & 581 & 24 \\
\hline 44 & Sui & 581 & 618 & 37 \\
\hline 45 & Tang & 618 & 907 & 289 \\
\hline 46 & Hou Liang & 907 & 923 & 16 \\
\hline 47 & Hou Tang & 923 & 936 & 13 \\
\hline 48 & Hou Jin & 936 & 947 & 11 \\
\hline 49 & Hou Han & 947 & 950 & 3 \\
\hline 50 & Hou Zhou & 951 & 960 & 9 \\
\hline 51 & Qian Shu & 891 & 925 & 34 \\
\hline 52 & Hou Shu & 925 & 965 & 40 \\
\hline 53 & Yang Wu & 892 & 937 & 45 \\
\hline 54 & Nan Tang & 937 & 975 & 38 \\
\hline 55 & Wu Yue & 893 & 978 & 85 \\
\hline 56 & Min Guo & 893 & 945 & 52 \\
\hline 57 & Ma Chu & 896 & 951 & 55 \\
\hline 58 & Nan Han & 905 & 971 & 66 \\
\hline 59 & Nan Ping & 907 & 963 & 56 \\
\hline 60 & Bei Han & 951 & 979 & 28 \\
\hline 61 & Bei Song & 960 & 1127 & 167 \\
\hline 62 & Nan Song & 1127 & 1279 & 152 \\
\hline 63 & Liao & 907 & 1125 & 218 \\
\hline 64 & $\mathrm{Da} \mathrm{Li}$ & 937 & 1254 & 317 \\
\hline 65 & Xi Xia & 1032 & 1227 & 195 \\
\hline 66 & Jin & 1115 & 1234 & 119 \\
\hline 67 & Yuan & 1206 & 1368 & 162 \\
\hline 68 & Ming & 1368 & 1644 & 276 \\
\hline 69 & Qing & 1616 & 1911 & 295 \\
\hline 70 & Republic of China & 1912 & 1949 & 37 \\
\hline
\end{tabular}

See notes in Table 1. This table is the second part of all Chinese dynasties. 


\subsection{Model results}

We use the survival time date shown in Tables 1 and 2 to estimate the Coxian phase-type model of Chinese dynasties shown in Figure 2. Model parameters are estimated by maximizing the log-likelihood function, which is given by Equation (8). Table 3 reports the estimated parameters results for the Coxian phase type model for the Chinese dynasties. All estimated parameters are significant at $5 \%$ level, except $\mu_{2}$.

Table 3: Model estimation results.

\begin{tabular}{lll}
\hline Parameter & Estimated results & Standard errors \\
\hline$\lambda$ & $0.0077^{* *}$ & 0.0026 \\
$\mu_{1}$ & $0.0202^{* *}$ & 0.0038 \\
$\mu_{2}$ & 0.0007 & 0.0033 \\
$\mu_{3}$ & $0.0108^{* *}$ & 0.0080 \\
\hline
\end{tabular}

Notes: This table reports the maximum log-likelihood estimates and the standard deviations for the parameters of the proposed Coxian phase-type model defined in Figure 2 . The asterisks $\left(^{* *}\right)$ represent significance at the $5 \%$ level.

The estimated parameters results indicate that the continuous-time Markov chain has the following infinitesimal generator matrix:

$$
\boldsymbol{Q}=\left(\begin{array}{ccc|c}
-0.0279 & 0.0077 & 0 & 0.0202 \\
0 & -0.0084 & 0.0077 & 0.0007 \\
0 & 0 & -0.0108 & 0.0108 \\
\hline 0 & 0 & 0 & 0
\end{array}\right) .
$$

By comparing with Equations (11) and (12), we obtain the transition rate matrix $\boldsymbol{\Lambda}$ and the absorbtion rate vector $\boldsymbol{q}$, respectively. The estimated parameters present the transition rates in the Markov chain. Each off-diagonal entry indicates the rate of moving from one state to another state, and the diagnose entry shows the rate of leaving a specific state.

In our model, we interpret the meaning of each parameter shown in the $Q$-matrix (Equation (13)) as follows. Note that our model starts from the establishment state. Based on the theory of Markov chain (see, Norris (1997)), the establishment state takes two different independent exponential times $T_{1} \sim E(0.0077)$ and $T_{2} \sim E(0.0202)$, where $E(\delta)$ denotes the exponential distribution with the parameter $\delta>0$. If $T_{1}$ is smaller, the state of the Chinese dynasty transfers from the establishment state to the prosperity state; However, if $T_{2}$ is smaller, the Chinese dynasty is directly terminated. Furthermore, the rates associated with each state may be used to determine the probability of jumping between states. The time spent in the establishment state is exponentially distributed with the parameter $0.0077+0.0202=$ 0.0279. Therefore, the probability of jumping from the establishment state to the prosperity state is $0.0077 / 0.0279 \approx 0.2759$, implying the probability that a Chinese dynasty could develop and survive after the dynasty is established. On the other hand, the probability that a new established dynasty turns perished is 0.7241 . We notice that 0.7241 is much larger than 0.2759 , which is consistent with the 
Chinese history. This empirical result implies that a new dynasty is often built by overwhelming the previous dynasty and has to face various challenges from the remaining forces of the overthrown dynasty. Similarly, when the dynasty is in the prosperity state, the probability that the dynasty becomes into the rottenness state is $0.0077 / 0.0084 \approx 0.9167$ and the probability that the dynasty changes into the perishment state is 0.0833 . This empirical result indicates that when a Chinese dynasty is in its prosperity state, it is nearly impossible to make the regime of the dynasty come to an end. Hence, when a Chinese dynasty is in the prosperity state, foreign policy-makers may need reconsider their foreign affairs with China and business companies may consider long-term business cooperations with China.

The estimated parameters in the infinitesimal generator matrix $\boldsymbol{Q}$ can be used to calculate the average transition time between states. By using the property of the exponential distribution, it takes about $1 / 0.0077 \approx 130$ years on average for a dynasty transforming from the establishment state to the prosperity state. This empirical result indicates that a Chinese dynasty, whose political system is centralization of power, requires a long time period to develop in order to transit the society into the prosperity state. Moreover, this result also agrees with the typical Chinese history. For example, the "last empire" in China, Qing Dynasty, took about 134 years to achieve the so-called "Kangxi and Qianlong Flourishing Age", which is from 1661 to 1785 and widely known in the Chinese people. Similarly, when the dynasty is in the prosperity state, it takes the dynasty another $1 / 0.0077 \approx 130$ years moving from the prosperity state to the rottenness state. Besides, we find that the estimated absorbtion rates $\mu_{1}, \mu_{2}$ and $\mu_{3}$ are different, which indicates that Chinese dynasties have different death rates at different social states. The estimated parameter $\mu_{1}$ indicates that the dynasty can survive $1 / 0.0202 \approx 50$ years on average directly changing from the establishment state to the perishment state. The parameter $\mu_{2}$ shows that it takes the dynasty $1 / 0.0007 \approx 1439$ years transferring from the prosperity state to the perishment state. This long time period is in agreement with the Chinese history and the previous analysis. Finally, if the dynasty is in the rottenness state, it averagely needs $1 / 0.0108 \approx 93$ years to perish. This estimated result implies that even a rottenness Chinese dynasty still requires almost a century to disappear and that ordinary people in the rottenness state have to suffer. Based on the above analysis, policy-makers and business companies may design suitable framework of policies and business plans corresponding to the different average state transition time period in order to profit and cooperation maximization.

\section{Further analysis}

Our analysis is based on the estimated lifetime model for Chinese dynasties shown in the previous section. With our fitted Coxian phase-type model, many variables of interest can be quantified. We are interested in revealing the developing trends and social state transitions properties for the Chinese dynasties. In this section, we measure different variables to characterize properties of the lifetime mechanism of Chinese dynasties.

\subsection{Transition probabilities}

Our dynasty model is based on the continuous-time Markov chain $\left\{X_{t}, t \geq 0\right\}$ with four different states shown in Figure 2. The four states represent four different and typical social states for Chinese 
dynasties. Hence, it is natural to quantify transition probabilities between these four states. Given the estimated infinitesimal generator matrix $\boldsymbol{Q}$ of the developing dynasty process $X(t)$, quantifying the transition probabilities between these states has important implication for risk management and formulating policies.

The estimated infinitesimal generator matrix $\boldsymbol{Q}$ for our dynasty model is given by Equation (13). To determine the transition probabilities between four social states, we use the Kolmogorov forward equation. Let the transition probability from the state $i$ to the state $j$ denote as

$$
p_{i j}(t)=\mathbb{P}\left(X_{h+t}=j \mid X_{h}=i\right) .
$$

The transition probabilities $p_{i j}(t)$ compose the transition probability matrix and can be calculated explicitly. Let $\boldsymbol{P}(t)$ denote the transition probability matrix for the continuous-time Markov chain $X(t)$. Then, the Kolmogorov forward equation is written as

$$
\boldsymbol{P}^{\prime}(t)=\boldsymbol{Q P}(t)
$$

and the initial condition for this equation is $\boldsymbol{P}(0)=\boldsymbol{I}$. Solving Equation (14) leads to

$$
\boldsymbol{P}(t)=e^{t \boldsymbol{Q}} .
$$

Therefore, with the estimated infinitesimal generator matrix $\boldsymbol{Q}$, we obtain the following transition probabilities between four different social states for Chinese dynasties:

1. $\mathbb{P}$ (establishment state in year $h+t \mid$ establishment state in year $h$ )

$$
p_{11}(t)=\exp (-0.0279 t)
$$

2. $\mathbb{P}$ (prosperity state in year $h+t \mid$ establishment state in year $h$ )

$$
p_{12}(t)=0.3949 \exp (-0.0084 t)-0.3949 \exp (-0.0279 t) .
$$

3. $\mathbb{P}($ rottenness state in year $h+t \mid$ establishment state in year $h$ )

$$
p_{13}(t)=1.267 \exp (-0.0084 t)-1.445 \exp (-0.0108 t)+0.1778 \exp (-0.0279 t) .
$$

4. $\mathbb{P}$ (perishment state in year $h+t \mid$ establishment state in year $h$ )

$$
p_{14}(t)=1.445 \exp (-0.0108 t)-1.662 \exp (-0.0084 t)-0.7829 \exp (-0.0279 t)+1
$$

5. $\mathbb{P}$ (prosperity state in year $h+t \mid$ prosperity state in year $h$ )

$$
p_{22}(t)=\exp (-0.0084 t)
$$

6. $\mathbb{P}($ rottenness state in year $h+t \mid$ prosperity state in year $h$ )

$$
p_{23}(t)=3.208 \exp (-0.0084 t)-3.208 \exp (-0.0108 t)
$$


7. $\mathbb{P}$ (perishment state in year $h+t \mid$ prosperity state in year $h$ )

$$
p_{24}(t)=3.208 \exp (-0.0108 t)-4.208 \exp (-0.0084 t)+1 \text {. }
$$

8. $\mathbb{P}$ (rottenness state in year $h+t \mid$ rottenness state in year $h$ )

$$
p_{33}(t)=\exp (-0.0108 t)
$$

9. $\mathbb{P}$ (perishment state in year $h+t \mid$ rottenness state in year $h$ )

$$
p_{34}(t)=1-\exp (-0.0108 t)
$$

10. $\mathbb{P}$ (perishment state in year $h+t \mid$ perishment state in year $h$ )

$$
p_{44}(t)=1
$$

11. Other transition probabilities are equal to zero.

These above transition probabilities may be used to predict the social states in Chinese dynasties and provide a quantitative perspective on making suitable regulations and foreign policies. For example, if a Chinese dynasty has already existed eighty years, we have the following explicit transition probabilities between the four different social states

$$
\boldsymbol{P}(80)=\left(\begin{array}{cccc}
0.1073 & 0.1593 & 0.0572 & 0.6762 \\
0 & 0.5107 & 0.2862 & 0.2031 \\
0 & 0 & 0.4215 & 0.5785 \\
0 & 0 & 0 & 1
\end{array}\right) .
$$

Take two elements as an example. The probability $\boldsymbol{P}_{11}(80)=0.1073$ means that after 80 years, the dynasty is still in the establishment state given that the dynasty is in the establishment state. The probability $\boldsymbol{P}_{14}(80)=0.6762$ implies that after 80 years, the dynasty comes into the perishment state given that the dynasty is in the establishment state. Hence, policy-makers may use these probabilities to make inference about social status to make policies and business companies may consider whether transfer their assets overseas to avoid risk. To sum up, computing transition probabilities provide useful information to identify the social state of Chinese dynasties.

\subsection{Hitting times and absorption probabilities}

Hitting times and absorption probabilities play an important role in the theory of Markov chain, see Norris (1997). For our lifetime mechanism of Chinese dynasties, hitting time refers to the theoretical time length to hit a certain social state, while absorption probabilities provide information about the probability that a Chinese dynasty perishes from a specific social state.

We first introduce definitions of hitting times and absorption probabilities, and then evaluate these variables for our dynasty model. Let $\{X(t), t \geq 0\}$ be a continuous-time Markov chain with the infinitesimal generator matrix $\boldsymbol{Q}$. The hitting time of a subset $A$ of the states of the process $X(t)$ is the random variable $D^{A}$ defined by

$$
D^{A}(\omega)=\inf \left\{t \geq 0: X_{t}(\omega) \in A\right\}
$$


with $\inf \varnothing=\infty$. The probability, starting from $i$, that $X(t)$ ever hits $A$ is then

$$
h_{i}^{A}=\mathbb{P}_{i}\left(D^{A}<\infty\right)
$$

When $A$ is a closed class, $h_{i}^{A}$ is called the absorption probability. The average time taken for $X(t)$ to reach $A$ is given by

$$
k_{i}^{A}=\mathbb{E}\left(D^{A}\right)
$$

It can be shown that (see, Norris (1997)) $h_{i}^{A}$ is satisfied with

$$
\begin{array}{r}
h_{i}^{A}=1 \text { for } i \in A \\
\sum_{j} q_{i j} h_{j}^{A}=0 \text { for } i \notin A
\end{array}
$$

and that $k_{i}^{A}$ is satisfied with

$$
\begin{array}{r}
k_{i}^{A}=0 \text { for } i \in A \\
-\sum_{j} q_{i j} k_{j}^{A}=1 \text { for } i \notin A .
\end{array}
$$

These equations can be used to compute $h_{i}^{A}$ and $k_{i}^{A}$, respectively. To better interpret the meaning of $h_{i}^{A}$ and $k_{i}^{A}$ for our dynasty model, we write less formally

$$
\begin{aligned}
h_{i}^{A} & =\mathbb{P}(\text { hit } A) \\
k_{i}^{A} & =\mathbb{E}(\text { time to hit } A) .
\end{aligned}
$$

Let $A$ be the perishment phase in our model and write $h_{i}^{A}$ and $k_{i}^{A}$ as $h_{i}$ and $k_{i}$ for simplicity. It is very interesting to calculate the absorption probability $h_{i}$ and the arrival average time $k_{i}$ for our dynasty model, because, $h_{i}$ indicates the probability that a Chinese dynasty enter into the perishment phase, and $k_{i}$ shows the average time to arrive the perishment phase for a Chinese dynasty.

Given the estimated infinitesimal generator matrix $\boldsymbol{Q}$ (Equation (13)), we evaluate $h_{i}$ and $k_{i}$ to further determine the mathematical mechanism of Chinese dynasties, respectively. We first compute $h_{i}$. In our model there is only one absorbing phase, which is the perishment state, indicating that each dynasty finally is bounded to be destroyed. Hence, we have the probability that enters into the absorbtion state is 1 , namely, $h_{1}=h_{2}=h_{3}=h_{4}=1$. This result indicates that each Chinese dynasty eventually perish. Then, a new dynasty is built up, and repeats the same pattern. Then, we compute $k_{i}$. By solving linear equations for Equations (15) and (16):

$$
\begin{aligned}
\boldsymbol{Q}_{11} k_{1}+\boldsymbol{Q}_{12} k_{2}+\boldsymbol{Q}_{13} k_{3}+\boldsymbol{Q}_{14} k_{4} & =-1 \\
\boldsymbol{Q}_{21} k_{1}+\boldsymbol{Q}_{22} k_{2}+\boldsymbol{Q}_{23} k_{3}+\boldsymbol{Q}_{24} k_{4} & =-1 \\
\boldsymbol{Q}_{31} k_{1}+\boldsymbol{Q}_{32} k_{2}+\boldsymbol{Q}_{33} k_{3}+\boldsymbol{Q}_{34} k_{4} & =-1 \\
k_{4} & =0
\end{aligned}
$$


we have that

$$
\begin{aligned}
& k_{1}=92.1224 \\
& k_{2}=203.9242 \\
& k_{3}=92.5926
\end{aligned}
$$

The solution for $k_{1}$ indicates that when a Chinese dynasty is in the establishment phase, on average, it takes about 92 years to perish. The solution for $k_{2}$ implies that when a Chinese dynasty is in the prosperity phase, the meaning time to perish is approximated 203 years. Finally, the solution for $k_{3}$ shows that when a Chinese dynasty is in the rottenness phase, the expected time to perish takes nearly 92 years. These solutions for average time to perish are consistent with the history of China. For policy-makers and companies, these solutions provide information to manage political risk. Following the average time length for hitting the absorbtion state, they may fully prepare directions of deep-cooperations with different Chinese dynasties.

\subsection{Survival function}

For our dynasty model, the survival function measures the probability that a dynasty would survive after existing several years. Let the random variable $T$ denote the time from the establishment of the dynasty to its perishment, then $T$ follows a Coxian phase-type distribution with the representation $(\boldsymbol{\alpha}, \boldsymbol{\Lambda})$. More specifical, the estimated $\boldsymbol{\alpha}$ and $\boldsymbol{\Lambda})$ are obtained from the estimated infinitesimal generator matrix $\boldsymbol{Q}$ (Equation (13)).

Following the mathematical properties of phase-type distribution (Equation (4)), we find that the survival function $S(t)$ of $T$ is of the following closed-form:

$$
S(t)=1.662 \exp (-0.0084 t)-1.445 \exp (-0.0108 t)+0.7829 \exp (-0.0279 t) .
$$

The survival function $S(t)$ shows the probability that a Chinese dynasty will survive after surviving $t$. Figure 3 plots the estimated survival function $S(t)$ (Equation (17)) for Chinese dynasties. It can be seen that $S(t)$ decreases monotonously and the survival probability of Chinese dynasties decreases significantly after surviving 100 years. The survival probability that the Chinese dynasties exist 100 years is about 0.27 . We consider the 100-years survival probability because there are less dynasties living beyond 100 years in the Chinese history. The survival probability that the Chinese dynasties exist 300 years is about 0.07 . We note this probability because only a few well-known historical Chinese dynasties can survive about 300 years. The survival probability that the Chinese dynasties exist 600 years is about 0.008. We calculate this probability because the longest surviving time for the Chinese dynasties is 646 years. These empirical results indicate that all the Chinese dynasties only have limited surviving time periods. However, the Chinese history shows that every emperor is keen to make his dynasty long live generation after generation so that his family could inherit the supreme power forever.

We can use the random variable $T$ to compute the mean time to failure by considering its expectation. In our model, the mean time to failure shows the average time before the dynasty moves into the perishment state. The mean time to failure is computed as

$$
\mathbb{E}(T)=-\boldsymbol{\alpha} \boldsymbol{\Lambda}^{-1} \boldsymbol{e} .
$$




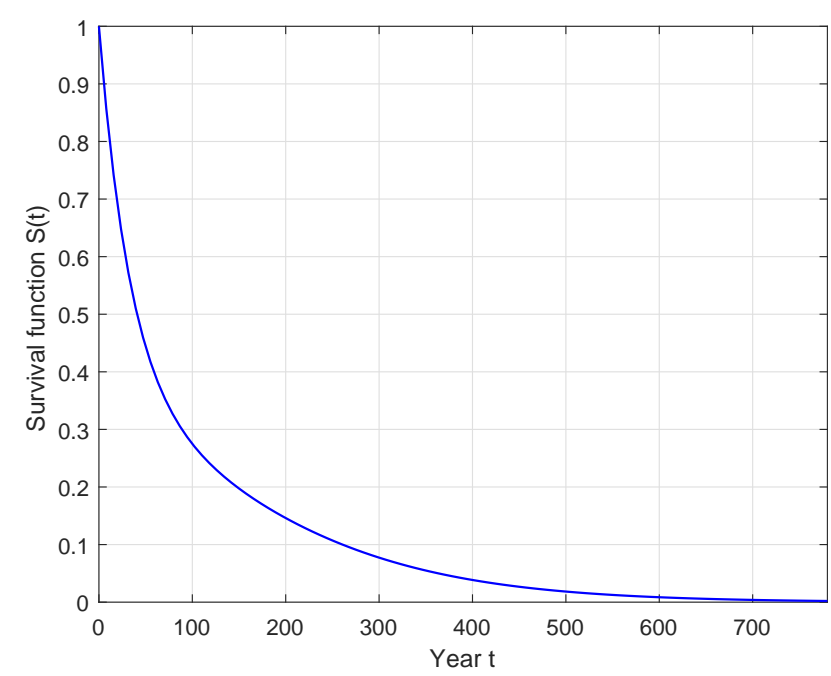

Figure 3: The survival function $S(t)$ for the Chinese dynasty estimated from the proposed dynasty model shown in Figure 2.

Using Equation (18) gives 92.1224 years, which is consistent with the solution $k_{1}$. To sum up, the survival function provides information about the survival time for predicting the existence time of Chinese dynasties.

\subsection{Phase analysis}

Conditional on the Chinese dynasty's already existing years, it is very interesting to identify the present social state for the Chinese dynasties. To analyze our proposed Chinese dynasty model, let

$$
\xi_{i}(t)=\mathbb{P}(X(t)=i \mid T>t)
$$

where $i$ is equal to 1,2 , and 3 , corresponding to the establishment state, the prosperity state, and the rottenness state, respectively. The definition of $\xi_{i}(t)$ is the probability that the dynasty is at state $i$ conditional on that the dynasty lives longer than time $t$. The probability $\xi_{i}(t)$ may be used to identify the current social state for Chinese dynasties and provides information for policy-makers. Equation (19) can be written as

$$
\xi_{i}(t)=\frac{\mathbb{P}(X(t)=i, T>t)}{\mathbb{P}(T>t)},
$$

where $\mathbb{P}(X(t)=i, T>t)$ is the probability that the social state $i$ survives longer than $t$ and is equal to $\left[\boldsymbol{\alpha} e^{\boldsymbol{\Lambda} t}\right]_{i}$, where $[\cdot]_{t}$ is the $i$ th component of vector $[\cdot]$, and $\mathbb{P}(T>t)$ is the survival function $S(t)$ for the random variable $T$. Note that $\sum_{i=1}^{3} \xi_{i}(t)=1$, implying that the Chinese dynasties stay in one of the social states before perishing.

We use the estimated results to approximate $\xi_{i}(t)$ through $\boldsymbol{\Lambda}$ and $S(t)$, which are already computed by Equations (13) and (17), respectively. WHence, we calculate $\xi_{i}(t)$ for the proposed Coxian phase-type model and obtain the following results:

1. $\xi_{1}(t)$ is the probability that the dynasty stays in the establishment state after living for $t$ years:

$$
\xi_{1}(t)=\frac{\exp (-0.0279 t)}{1.662 \exp (-0.0084 t)-1.445 \exp (-0.0108 t)+0.7829 \exp (-0.0279 t)} .
$$


2. $\xi_{2}(t)$ denotes the probability that the dynasty is in the prosperity state after living for $t$ years:

$$
\xi_{2}(t)=\frac{0.3949 \exp (-0.0084 t)-0.3949 \exp (-0.0279 t)}{1.662 \exp (-0.0084 t)-1.445 \exp (-0.0108 t)+0.7829 \exp (-0.0279 t)} .
$$

3. $\xi_{3}(t)$ presents the probability that the dynasty stays in the rootlessness state after $t$ years:

$$
\xi_{3}(t)=\frac{1.267 \exp (-0.0084 t)-1.445 \exp (-0.0108 t)+0.1778 \exp (-0.0279 t)}{1.662 \exp (-0.0084 t)-1.445 \exp (-0.0108 t)+0.7829 \exp (-0.0279 t)} .
$$

The values for $\xi_{i}(t)$ may be useful to identify the development for Chinese dynasties. Figure 4 plots $\xi_{i}(t)$ for $i=1,2,3$. It can be seen that $\xi_{i}(t)$ displays different behaviours and has different magnitude. We find that $\xi_{1}(t)$ decreases monotonically, implying that the Chinese dynasties cannot always stay in the establishment state. The values of $\xi_{2}(t)$ and $\xi_{3}(t)$ increase from zero, indicating that the probability that a Chinese dynasty enters into either the prosperity state or the rottenness state tends to increase. Comparing the values of $\xi_{2}(t)$ and $\xi_{3}(t)$ before 200 years, we find that $\xi_{2}(t)$ is slightly larger than $\xi_{3}(t)$, which may indicate that the Chinese dynasty tends to enter into the prosperity state. However, after 200 years, both $\xi_{2}(t)$ and $\xi_{3}(t)$ tend to be a stable value and $\xi_{2}(t)$ gradually becomes smaller than $\xi_{3}(t)$, implying that the traditional Chinese dynasties with the centralization power system finally move into the rottenness state. Note that when $t \rightarrow \infty, \xi_{1}(t) \rightarrow 0, \xi_{2}(t) \rightarrow 0.2394$ and $\xi_{3}(t) \rightarrow 0.7606$. These limited results imply that if a Chinese dynasty lives long enough, it is bound to lead to the rottenness state, which means that the dynasty are to perish in the end. This analysis is consistent with the history of China. The rottenness dynasties always are replaced by another new dynasty.

To illustrate the implication of $\xi_{i}(t)$, we consider an example. Figure 4 also displays red lines to show the probability of being in different states for a Chinese dynasty at year $t=100$. In specific, $\xi_{1}(100)=0.2235, \xi_{2}(100)=0.5322$ and $\xi_{1}(100)=0.2443$. These three values indicate that after 100 years of development, the state of a Chinese dynasty seems most likely to be the prosperity state, because $\xi_{2}(100)$ is the greates one. To sum up, by computing $\xi_{i}(t)$ from our proposed dynasty model, policymakers and business companies may consider to use $\xi_{i}(t)$ to identify the social state of Chinese dynasties in order to determine the proper foreign policies and investment strategies with Chinese dynasties.

\subsection{The force of mortality}

The concept of the force of mortality plays an important role in the theory of life contingencies and actuarial science. The force of mortality is a continuous function of age and is defined as the instantaneous effect of mortality at a certain age. Hence, it is also interesting to examine the force of mortality for Chinese dynasties. In this paper, the force of mortality for Chinese dynasties can be defined as the instantaneous mortality rate. The force of mortality $\gamma(t)$ is computed as

$$
\gamma(t)=\frac{\boldsymbol{\alpha} \exp (\boldsymbol{\Lambda} t) \boldsymbol{q}}{\boldsymbol{\alpha} \exp (\boldsymbol{\Lambda} t) \boldsymbol{e}}
$$

where the upper is the probability density function of the random variable $T$ given by Equation (2), and the bottom is the survival function of $T$ given by Equation (4).

Using the empirical result for $\boldsymbol{\Lambda}$, we evaluate $\gamma(t)$ for Chinese dynasties, which is shown in Figure 5. The force of mortality decreases quickly after a Chinese dynasty is established, and reaches its lowest 


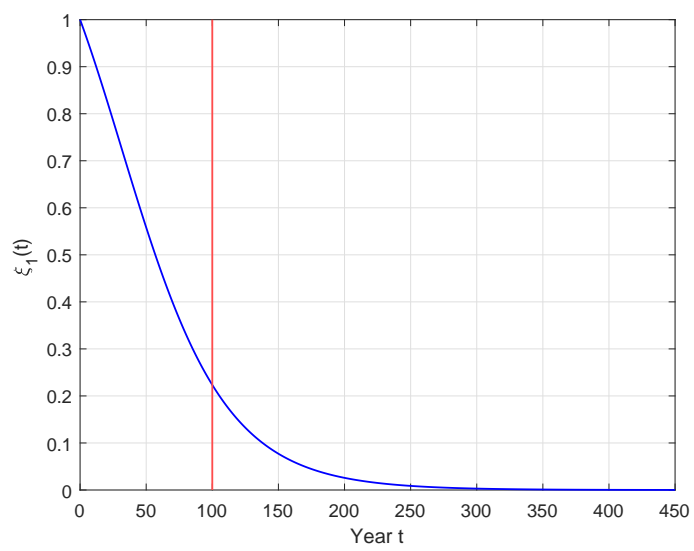

(a) The probability $\xi_{1}(t)$

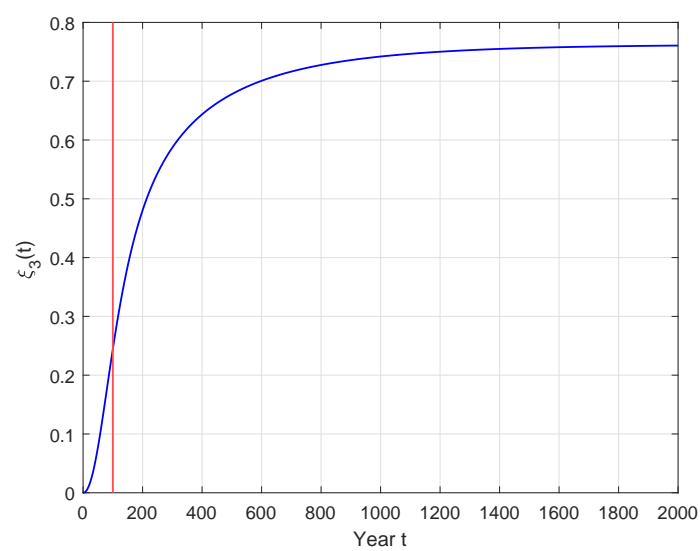

(c) The probability $\xi_{3}(t)$

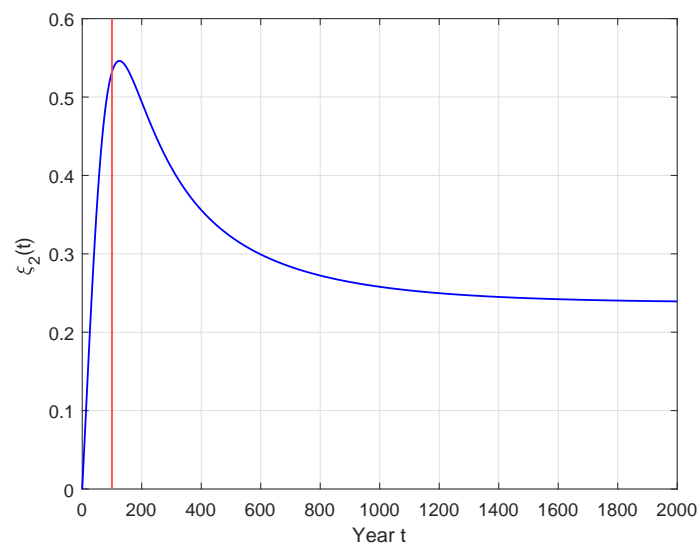

(b) The probability $\xi_{2}(t)$

Figure 4: $\xi_{i}(t)$ is the probability that the dynasty is at state $i$ conditional on that the dynasty lives longer than time $t$.

point after about 150 years. Then, the force of mortality slightly increases and starts to gradually level off as the risk of dying remains approximately constant, forming the so-called mortality plateau (see Pascariu and Torres (2020)). Note that such behavior of the mortality curve at advanced years is based on scarce data, as the number of Chinese dynasties reaching very long periods was relatively small according to the Chinese history. Furthermore, we find that the value of $\gamma(t)$ is very small, indicating that a dynasty at year $t$ perishes slowly. That is, the Chinese dynasty seems not become extinct suddenly. For policy-makers and business companies, they may consider the long term corporation with Chinese dynasties. 


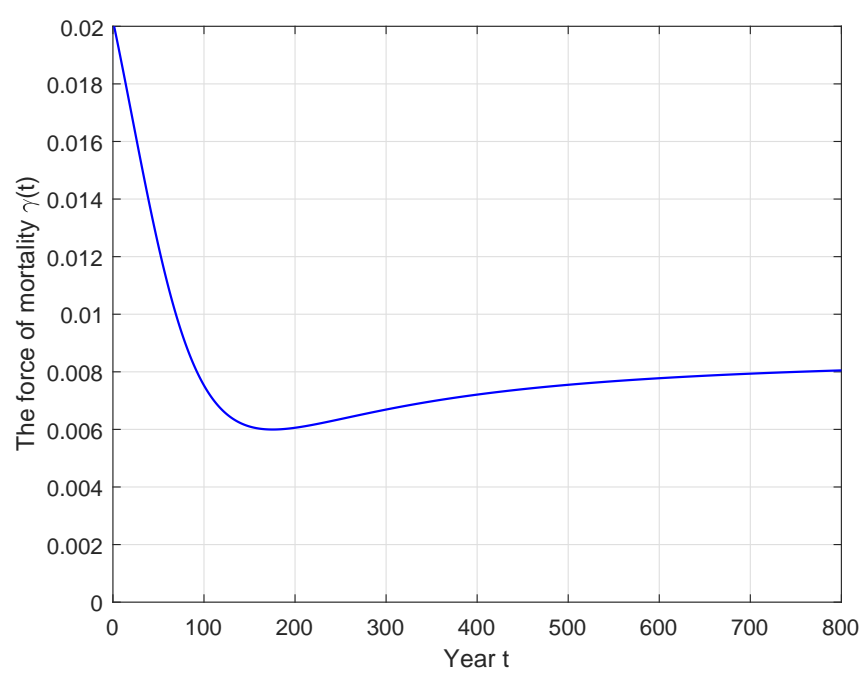

Figure 5: The force of mortality $\gamma(t)$ for Chinese dynasties.

\section{Conclusions and further work}

China has a long history and is composed of different and continuous dynasties. Almost all the Chinese dynasties undergo the process from establishment to perishment, and repeat this pattern circularly. Hence, it is interesting to investigate the development pattern of Chinese dynasties.

This paper proposes a mathematical model for the lifetime mechanism of the Chinese dynasties, and evaluates various properties for the Chinese dynasties. This model is associated with the continuous-time Markov chain and the Coxian phase-type distribution. Based on the feature of the Chinese dynasties, this paper assumes that the continuous-time Markov chain with four different social states, which are the establishment state, the prosperity state, the rottenness state and the perishment state. Furthermore, we estimate the Coxian phase-type distribution by maximizing the log-likelihood function.

With our estimated Coxian phase-type model, we calculate the variables which are closely related with the existing time for Chinese dynasties. These variables include transition probabilities between different social states, hitting times and absorption probabilities, the survival function, phase analysis and the force of mortality. Moreover, all these variables are expressed in the closed-forms. Hence, for policy-makers and business companies, these variables can be used to formulate foreign policies and business plans with Chinese dynasties and predict the social states of Chinese dynasties.

Following the lifetime model for traditional Chinese dynasties presented in this paper, the future work may consider to determine the ruling time for each Chinese emperor.

\section{Declaration of interests statement}

The authors declare no conflict of interest. 


\section{References}

Asmussen, S., 2003. Applied Probability and Queues. 2 ed., Springer, New York, NY. URL: https: //doi.org/10.1007/b97236, doi:10.1007/b97236.

Bladt, M., 2005. A review on phase-type distributions and their use in risk theory. ASTIN Bulletin 35, 145-161. URL: https://doi.org/10.1017/s0515036100014100, doi:10.1017/s0515036100014100.

Chan, K.S., Laffargue, J.P., 2016. A DYNAMIC MODEL OF TAXATION, CORRUPTION, AND PUBLIC INVESTMENT IN THE DYNASTIC CYCLE: THE CASE OF IMPERIAL CHINA. Macroeconomic Dynamics 20, 2123-2147. URL: https://doi.org/10.1017/s1365100515000218, doi:10.1017/s1365100515000218.

Chu, C.Y.C., Lee, R.D., 1994. Famine, revolt, and the dynastic cycle. Journal of Population Economics 7, 351-378. URL: https://doi .org/10.1007/bf00161472, doi:10.1007/bf00161472.

Cox, D.R., 1955. A use of complex probabilities in the theory of stochastic processes. Mathematical Proceedings of the Cambridge Philosophical Society 51, 313-319. URL: https://doi .org/10.1017/ s0305004100030231, doi:10.1017/s0305004100030231.

Faddy, M., 1998. On inferring the number of phases in a coxian phase-type distribution. Communications in Statistics. Stochastic Models 14, 407-417. URL: https://doi.org/10.1080/15326349808807479, doi:10.1080/15326349808807479.

wai Fan, K., 2009. Climatic change and dynastic cycles in chinese history: a review essay. Climatic Change 101, 565-573. URL: https://doi.org/10.1007/s10584-009-9702-3, doi:10.1007/ s10584-009-9702-3.

Govorun, M., Jones, B.L., Liu, X., Stanford, D.A., 2018. Physiological age, health costs, and their interrelation. North American Actuarial Journal 22, 323-340. URL: https://doi.org/10.1080/ 10920277.2017 .1404476 , doi:10.1080/10920277.2017.1404476.

Higham, N.J., 2008. Functions of Matrices: Theory and Computation. Society for Industrial and Applied Mathematics. URL: https://doi.org/10.1137/1.9780898717778, doi:10.1137/1.9780898717778.

Hobolth, A., Siri-Jégousse, A., Bladt, M., 2019. Phase-type distributions in population genetics. Theoretical Population Biology 127, 16-32. URL: https://doi.org/10.1016/j.tpb.2019.02.001, doi:10.1016/j.tpb.2019.02.001.

Luo, G., 2004. Three Kingdoms: A Historical Novel. 1st ed., University of California Press. URL: https : //www . ucpress.edu/book/9780520224780/three-kingdoms-a-historical-novel\#about-book.

Neuts, M.F., 1981. Matrix-Geometric Solutions in Stochastic Models: An algorithmic approach. The John Hopkins University Press, Baltimore. 
Neuts, M.F., 1991. Structural stochastic matrices of m/g/1 type and their applications. Acta Applicandae Mathematicae 24, 205-206. URL: https://doi.org/10.1007/bf00046899, doi:10.1007/ bf 00046899 .

Norris, J.R., 1997. Markov Chains. Cambridge University Press. URL: https://doi.org/10.1017/ cbo9780511810633, doi:10.1017/cbo9780511810633.

Okamura, H., Dohi, T., 2016. Fitting phase-type distributions and markovian arrival processes: Algorithms and tools, in: Springer Series in Reliability Engineering, pp. 49-75. URL: https: //doi.org/10.1007/978-3-319-30599-8_3, doi:10.1007/978-3-319-30599-8_3.

Pascariu, M.D., Torres, C., 2020. Force of mortality, in: Encyclopedia of Gerontology and Population Aging. Springer International Publishing, pp. 1-6. URL: https://doi.org/10.1007/ 978-3-319-69892-2_551-1, doi:10.1007/978-3-319-69892-2_551-1.

Payne, K., Marshall, A.H., Cairns, K.J., 2011. Investigating the efficiency of fitting coxian phase-type distributions to health care data. IMA Journal of Management Mathematics 23, 133-145. URL: https://doi.org/10.1093/imaman/dpr008, doi:10.1093/imaman/dpr008.

Skinner, G.W., 1985. Presidential address: The structure of chinese history. The Journal of Asian Studies 44, 271-292. URL: https://doi.org/10.2307/2055923, doi:10.2307/2055923.

Usher, D., 1989. The dynastic cycle and the stationary state. The American Economic Review 79, 1031-1044. URL: http://www.jstor.org/stable/1831435.

Yu-Ch'uan, W., 1936. The rise of land tax and the fall of dynasties in chinese history. Pacific Affairs 9, 201. URL: https://doi.org/10.2307/2751407, doi:10.2307/2751407.

Zhang, Q., 2015. An Introduction to Chinese History and Culture. Springer Berlin Heidelberg. URL: https://doi .org/10.1007/978-3-662-46482-3, doi:10.1007/978-3-662-46482-3. 